\title{
Impact of optical free-carrier generation on the performance of SOI phase shifters
}

\author{
Clemens J. Krückel $^{1,2}$, Joris Van Campenhout ${ }^{2}$, and Dries van Thourhout ${ }^{1,2}$ \\ ${ }^{1}$ Photonics Research Group, INTEC, Ghent University-IMEC, Ghent B-9000, Belgium \\ ${ }^{2}$ IMEC, Kapeldreef 75, Leuven B-3001, Belgium \\ clemens.kruckel@ugent.be
}

\begin{abstract}
We provide measurement and simulation data of optical free-carrier generation in SOI phase shifters. We conclude that phase impairments caused by unwanted freecarriers can be equalized with an $\sim 50 \%$ increase in phase shifter diode current. (C) 2020 The Author(s)
\end{abstract}

\section{Introduction}

In the EU-project SPICE [1], we work on the co-integration of photonics and spintronics with the objective to implement data storage based on spintronic memory. Spintronic memories are both non-volatile and high-speed and additionally they can be written all-optically [2], which offers reduced energy-consumption compared to electrical writing. We use photonic chips as the routing layer to address individual memory elements via grating-couplers. As a photonics platform silicon-on-insulator (SOI) is favorable. This platform allows the implementation of compact focusing-grating-couplers [3] and additionally the realization of optical routing networks. These networks can be implemented using electro-optic switches with phase shifters based on the plasma dispersion effect [4]. In [5] we showed the advantages of carrier-injection based phase shifters for our application. These components rely on the diffusive transport of charge carriers across the optical waveguide to induce an optical phase shift. As all-optical writing of spintronic memory still requires high optical intensities after the photonic chip, nonlinear carrier generation might take place in the phase shifters altering their phase state. Although nonlinear carrier generation has been studied in strip waveguides [6], the comprehensive characterization of carrier effects in SOI phase shifters is missing. Here, we study the impact of bulk and surface carrier generation on the performance of carrier-injection based phase shifters.

\section{Characterization of strip and rib waveguides}

First, we study reference waveguides without phase shifters in order to calibrate the simulation model. For this we characterize the attenuation induced by optically generated free-carriers. The reference systems have a length of around $3 \mathrm{~mm}$ and contain strip and rib waveguides with cross-section designs as shown in Fig. 1a. Both waveguides are used in the phase shifter systems presented below. For the measurements we gradually increase the continuous wave $(\mathrm{CW})$ power launched into the waveguide and quantify the optical power before and after the chip (Fig 1b). The numerical simulations of these waveguides are based on solving the nonlinear Schröedinger equation with the split-step method [7]. In every waveguide step carrier generation and correspondingly the free-carrier absorption (FCA) is calculated. Besides FCA, the simulations include linear loss (see Fig. 1a), two-photon absorption (TPA) $\left(\beta_{T P A}=1.5 \mathrm{~cm} / \mathrm{GW}[6]\right)$ and a coupling loss of $2.9 \mathrm{~dB}$. The simulations include both bulk free-carrier generation via TPA and surface free-carrier-generation via single photon absorption (SPA) [6]. In order to match measurements and simulations, we use carrier lifetime and the SPA coefficient as fitting parameters (see Fig. 1b for results). Additionally, Fig. 1c shows the simulated power propagation in the waveguide, here exemplified for an input power level of $23 \mathrm{dBm}$. The rib waveguide is separated from the input/output grating couplers by a 50nm long strip waveguide. This section causes increased optical attenuation as visible in the figure. A more than $5 \mathrm{~dB}$ higher output power of the rib waveguide systems makes this design more suitable for high power routing between phase shifter elements.

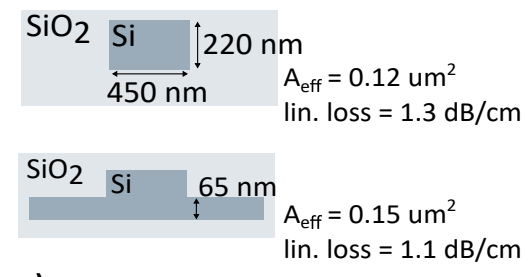

a)

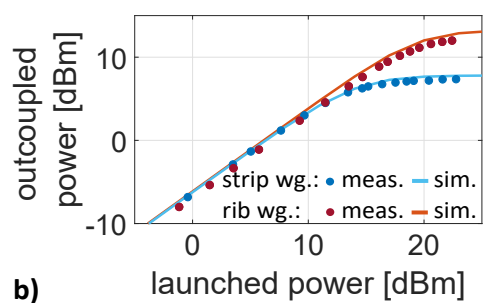

b)

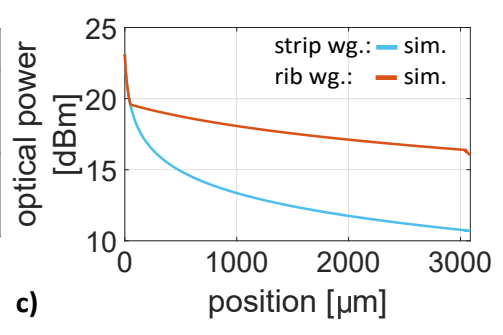

Fig. 1. a) Illustration of strip and rib waveguide cross-section. b) Measured and simulated power dependent system transmission. c) Length resolved optical power decay. 

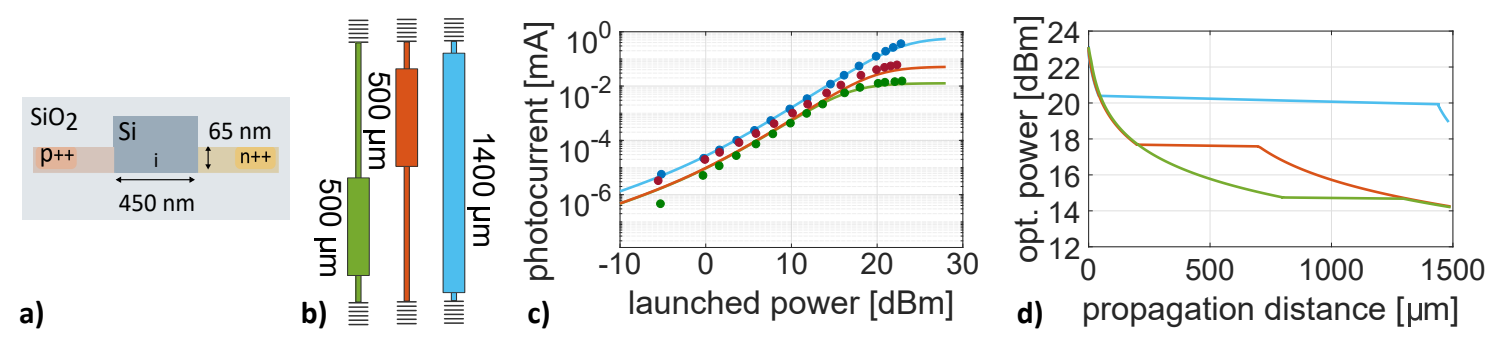

d) propagation distance $[\mu \mathrm{m}]$

Fig. 2. a) Illustration of pin-diode cross-section. b) Illustration of measured phase shifter systems. c) Results of measured and simulated photocurrent versus optical input power. d) Simulated e) Simulation of length resolved optical power decay.

\section{Characterization of phase shifter system and conclusion}

Next, we study in detail the carrier-injection based phase shifters with a design as shown in Fig. 2a. The phase shifter systems we used contain a phase shifter section bordered by strip waveguides that connect to grating couplers. Since phase shifter length and location in the system plays a significant role, we present three different diode arrangements illustrated in Fig. 2b, all with the same total system length of $1.5 \mathrm{~mm}$. Besides optical transmission, we furthermore measure the photocurrent from the unbiased pin-diode. Fig. 2c shows the photocurrent at various power levels. For the simulations we concatenate the three waveguide sections with their individual characteristics (e.g. $\left.A_{e f f}, \tau_{e f f}\right)$. We achieved a good match between measurement and simulations using free-carrier lifetime and the SPA coefficient as fitting parameters. The simulated power propagation in Fig. $2 d$ indicates that shorter access waveguides and a longer diode section results in a significantly higher output power. This is beneficial for our application but this comes along with equivalent higher carrier concentration in the diode. In order to illustrate the impact of these carriers on the optical phase shift in the diode, we take a closer look at the $1400 \mu \mathrm{m}$ long phase shifter. Fig. 3a shows the total amount of generated carriers in the diode versus input power. The simulations indicate that for launched power levels below $0 \mathrm{dBm}$ the main contribution to the free-carrier generation originates from surface states. According to the plasma dispersion relation [4], the phase shift scales with the amount of charge carriers as shown in Fig. 3b. Thus, with the simulated amount of carriers not exceeding $10^{-12}$, the expected phase shift caused by optical free-carrier generation is expected to be less than $0.25 \pi$. To compensate for this phase shift and to achieve a relative phase shift of $\pi$ with respect to the off-state, the diode current has to be adjusted by around $0.13 \mathrm{~mA}$ (see Fig. 3c).

In conclusion, these simulations imply that optically generated free-carriers impact the state of the phase shifter at launched power values above $14 \mathrm{dBm}$ when the optical free-carrier generation reaches $10^{-14}-10^{-13} \mathrm{C}$. The presented data give valuable insights in order to optimize a phase shifter network for the application of all-optical writing of magnetic-spintronic memory cells.


Fig. 3. a) Accumulated generated free-carriers in the $1400 \mu \mathrm{m}$ long diode region. TPA two-photon absorption. SPA single-photon absorption. b) Theoretical optical phase shift versus charge carriers. c) Measured optical phase shift versus diode current in a $1400 \mu \mathrm{m}$ pin-diode phase shifter.

Acknowledgements

This project has received funding from the European Union's Horizon 2020 research and innovation programme under grant agreement No 713481.

\section{References}

1. http://spice-fetopen.eu/

2. J.-Y. Chen et al., 'All-Optical Switching of Magnetic Tunnel Junctions ...', Phys. Rev. Appl., 2017, 7, (2), pp. 021001-6

3. H. Becker et al., "Out-of-Plane Focusing Grating Couplers for Silicon Photonics ...," JSTQE 26, (2), 8300408 (2020)

4. R. Soref et al., 'Electrooptical Effects in Silicon', IEEE J. Quantum Electron., 1987, 23, (6), pp. 123-129

5. C.J. Krückel et al., 'Towards High-Speed Energy-Efficient ...', CLEO Europe, Munich, Germany, June 2019

6. A. Gil-Molina et al., "Optical free-carrier generation in silicon ...," Appl. Phys. Lett. 112, 251104-2 (2018)

7. G.P. Agrawal, "Nonlinear Fiber Optics," Academic Press, 5th ed., (2013) 